\title{
A Variational Method for the Optimization of Tone Mapping Operators
}

\author{
Praveen Cyriac, Thomas Batard, and Marcelo Bertalmío \\ Department on Information and Communication Technologies, \\ Universitat Pompeu Fabra, Barcelona, Spain \\ \{praveen. cyriac, thomas . batard, marcelo. bertalmio\}@upf . edu
}

\begin{abstract}
Given any metric that compares images of different dynamic range, we propose a method to reduce their distance with respect to this metric. The key idea is to consider the metric as a non local operator. Then, we transform the problem of distance reduction into a non local variational problem. In this context, the low dynamic range image having the smallest distance with a given high dynamic range is the minimum of a suitable energy, and can be reached through a gradient descent algorithm. Dealing with an appropriate metric, we present an application to Tone Mapping Operator (TMO) optimization. We apply our gradient descent algorithm, where the initial conditions are Tone Mapped (TM) images. Experiments show that our algorithm does reduce the distance of the TM images with the high dynamic range source images, meaning that our method improves the corresponding TMOs.
\end{abstract}

Keywords: Tone mapping, Dynamic range independent metric, Contrast distortion, Variational methods.

\section{Introduction}

The vast range of light intensities of the real world span many orders of magnitude. The human visual system can handle intensities from about $10^{-6}$ to $10^{8}$ [18, because it continuously adjusts to the light in any viewed scene. High Dynamic Range (HDR) images constitute a powerful tool to store those intensities. However, they cannot be reproduced in common display devices and printers, as these usually span only two orders of magnitude. So an operation called "tone mapping" is performed on the HDR image to scale down the dynamic range in order to obtain a Low Dynamic Range (LDR) image. A good TMO should produce in the observer a sensation as close as possible to the perception produced by the real-world scene. In particular, it should neither introduce nor lose details.

As mentioned in Ferradans et al. [9, TMOs might be classified into two categories. There is a category of TMOs inspired by vision models, like Retinex theory [10, anchoring theory [1], Naka-Rushton formula ([18], 20], 12]), WeberFechner's law $(2], 25])$. There exists a second category, called gradient-based 
TMOs, which rely on the idea of shrinking large intensity gradients while preserving small fluctuations, corresponding to fine details $([6,6],[14,[23])$. We refer to [19] for more details about tone mapping and HDR imaging.

An accurate evaluation of TMOs requires a measure of comparison of color sensation and detail visibility between the source (an HDR image) and the output (an LDR image). To the best of our knowledge, there exists no perceptual based measure that compares color appearance between HDR and LDR images. On the other hand, metrics comparing detail visibility or contrast between HDR and LDR images have been investigated (see e.g. [3],[22]). In particular, an objective tone mapping evaluation tool has been proposed by Smith et al. [22], based on the measure of suprathreshold contrast distortion between the source HDR image and its TM LDR version. However, the contrast measure holds only for neighboring pixels, meaning that its sensitivity is limited to high frequency details. More recently, Aydin et al. 3] proposed a metric (DRIM) independent of the dynamic range whose contrast measure is not limited to neighboring pixels (see Sect. 3.1 for a description of the metric DRIM).

Our contribution is two-fold. We first introduce a general framework to reduce the distance (with respect to a given metric) between two images of any dynamic range. Then, we make use of this dynamic range independence to optimize any existing TMO with respect to a given metric. In this paper, we focus on the metric DRIM [3], however we would like to point out that our method is very general and may be applied to any metric. Considering the metric as a non local operator, we are led to a non local variational problem where the quantity to be minimized is a distance associated to the metric. We test our method on the TMOs of Drago et al. [5], Ferradans et al. 9], Mantiuk et al. [15, Reinhard et al. [20]. Experiments show that our method appreciably reduces contrast distortion in the results of the aforementioned TMOs.

The paper is organized as follows. In Sect. 2, we introduce the variational problem, that consists in minimizing a distance between images. We show that this approach is very generic since we only require the metric to be a non local operator. We discuss both continuous and discrete cases. In Sect. 3, we treat the special case where the metric involved is the metric DRIM. We first remind the main properties of this metric. Then, we provide applications to TMOs optimization. We detail a preprocessing that we apply on the TM image we aim at modifying. Finally, we show results of our minimization algorithm applied on the output of the preprocessing, and compare with the initial TM images.

\section{Minimization of the Distance between Images}

\subsection{Image Quality Metrics as Non Local Operators}

Many tasks in image processing and computer vision require a validation by comparing the result with the original data, e.g. optical flow estimation, image denoising, tone mapping. Whereas measures based on pixel-wise comparisons (e.g. MSE, SNR, PSNR) are suitable to evaluate image denoising and optical flow estimation algorithms, they are not adapted to evaluate tone mapping. 
Tone mapping evaluation requires a measure that compares color sensation and detail visibility (contrast), which are not pixel-wise neither local concepts. This leads us to the following definitions.

Definition 1 (metric). Let $L, H: \Omega \longrightarrow \mathbb{R}$ be two images. We call metric an operator met such that met $(L, H)$ is of the form $\operatorname{met}(L, H): \Omega \longrightarrow \mathbb{R}^{n}, n \geq 1$.

We say that a metric is non local if the quantities

$$
\frac{\partial \operatorname{met}(L, H)(x)}{\partial L(y)}, \frac{\partial \operatorname{met}(L, H)(x)}{\partial H(y)}
$$

do not vanish for some points $y \neq x$.

The two terms in (1) are infinite dimensional extensions of the notion of derivation with respect to the $n$-th component. For instance, the first term means that we compute the variations of the functional $L \longmapsto \operatorname{met}(L, H)(x)$ with respect to the variations of the function $L$ at the point $y$.

In this context, we can classify image quality measures into three categories. The first one is the class of metrics that are not non local, like MSE, PSNR and SNR measures. Indeed, the term (11) vanishes since such metrics are based on pixel-wise differences. The second category gathers the metrics that are non local and compare images of same dynamic range (see e.g. 4 for LDR images, [13, [16] for HDR images, and 24] for images of any dynamic range). The last category contains the metrics that are non local and compare images of different dynamic range (see e.g. 3], 22]). In particular, the metric DRIM [3] belongs to both second and third categories since it is independent of the dynamic range of the images it compares.

Definition 2 (distance between images). A distance associated to the metric met is an energy $E$ of the form

$$
E:(L, H) \longmapsto \frac{1}{|\Omega|} \int_{\Omega} \Phi(\operatorname{met}(L, H)(x)) d x
$$

where $\Phi: \mathbb{R}^{n} \longrightarrow \mathbb{R}^{+}$is a differentiable map.

\subsection{Minimization of the Distance: Continuous Formulation}

From now on, we assume that $H$ is fixed and met is a non local metric. We aim at finding the images $L$ that minimize the distance with $H$. A necessary condition for $L$ to be a minimum of the energy (2) is that $L$ is a critical point of (2), i.e. the differential of the energy at this point in every direction is 0 .

Proposition 1. The images $L$ which are critical points of the energy (2) satisfy

$$
\int_{\Omega} \partial \Phi\left(\operatorname{met}(L, H)(x) ; \frac{\partial \operatorname{met}(L, H)(x)}{\partial L(y)}\right) d x=0 \quad \forall y \in \Omega
$$


Proof. Let $\psi: \Omega \longrightarrow \mathbb{R}$ be a compact support function. We compute the differential $\partial E$ of the energy (2) at a point $(L, H)$ in the direction $(\psi, 0)$. We have

$$
\begin{aligned}
\partial E((L, H) ;(\psi, 0)) & =\frac{1}{|\Omega|} \int_{\Omega} \partial(\Phi \circ m e t)((L, H) ;(\psi, 0))(x) d x \\
& =\frac{1}{|\Omega|} \int_{\Omega} \partial \Phi(\operatorname{met}(L, H)(x) ; \partial \operatorname{met}((L, H) ;(\psi, 0))(x)) d x
\end{aligned}
$$

by the composition law in differential calculus.

Then,

$$
\partial \operatorname{met}((L, H) ;(\psi, 0)(x))=\int_{\Omega} \frac{\partial \operatorname{met}(L, H)(x)}{\partial L(y)} \psi(y) d y
$$

Finally we have

$$
\partial E((L, H) ;(\psi, 0))=\frac{1}{|\Omega|} \int_{\Omega} \int_{\Omega} \partial \Phi\left(\operatorname{met}(L, H)(x) ; \frac{\partial \operatorname{met}(L, H)(x)}{\partial L(y)}\right) \psi(y) d x d y
$$

At last, as $\psi$ has compact support, $\partial E((L, H) ;(\psi, 0))=0 \Longrightarrow$

$$
\int_{\Omega} \partial \Phi\left(\operatorname{met}(L, H)(x) ; \frac{\partial \operatorname{met}(L, H)(x)}{\partial L(y)}\right) d x=0 \quad \forall y \in \Omega
$$

The term

$$
y: \longmapsto \frac{1}{|\Omega|} \int_{\Omega} \partial \Phi\left(\operatorname{met}(L, H)(x) ; \frac{\partial \operatorname{met}(L, H)(x)}{\partial L(y)}\right) d x
$$

is the gradient $\nabla(E)$ of the functional $L: \longmapsto E(L, H)$.

It is not trivial to obtain mathematical properties of functionals (2) because of the nature of the operators met. Hence, it is hard to establish accurate numerical schemes in order to get the minima of the energy (2). For this reason, we deal in this paper with the gradient descent algorithm, which has the property of being applicable to a large class of functionals. Given an image $L_{0}$, we consider the scheme

$$
L_{k+1}=L_{k}-\alpha_{k} \nabla(E)\left(L_{k}\right), \quad L_{\mid t=0}=L_{0}
$$

However, such a method may reach critical points that are local minima and not global minima. Hence, the initial condition has to be carefully chosen.

\subsection{Minimization of the Distance: Discrete Formulation}

We assume that $L$ and $H$ are of size $M \times N$. Following Sect.2.2, we define the (discrete) distance between $L$ and $H$ as

$$
E(L, H)=\frac{1}{M N} \sum_{m=1}^{M} \sum_{n=1}^{N} \Phi(\operatorname{met}(L, H)(m, n))
$$

In this section, we construct a discrete counterpart of the gradient (5). The main task is to define a discrete counterpart of the term (11). In particular, the term $\partial L(y)$ yields the construction of pixel-wise intensity increments. 
Pixel-wise Intensity Increments. There is not a unique choice to compute pixel-wise intensity increments. The trivial approach consists in considering the value $L_{+}(i, j):=L(i, j)+k$ as forward increment and $L_{-}(i, j):=L(i, j)-k$ as backward increment at a pixel $(i, j)$, for a fixed $k \in \mathbb{R}^{+*}$. However we claim that such an approach is not adapted to the problem addressed here. Indeed, because the metric met in (11) involves perceptual concepts, we intuit that the increments should involve perceptual concepts too.

In [21, a perceptual distance between pixels that takes into account the Weber law principle has been constructed. In particular, the authors construct a "perceptual gradient"

$$
\nabla_{W} I:=\frac{\nabla I}{I}
$$

for image denoising purposes.

Then, we define the backward $L_{-}(i, j)$ and forward $L_{+}(i, j)$ increment at the pixel $(i, j)$ by requiring their perceptual distance (in the sense of Shen) with $L(i, j)$ to be equal, i.e.

$$
\frac{L_{+}(i, j)-L(i, j)}{L(i, j)}=\frac{L(i, j)-L_{-}(i, j)}{L(i, j)}=k
$$

for some $k>0$.

Expression of the Discrete Gradient. Given a constant $\lambda \in] 0,1[$, we construct for each pixel $(i, j)$ two images $L_{+}(i, j)$ and $L_{-}(i, j)$ defined as follows

$$
\begin{aligned}
& L_{+}(i, j):(m, n) \longmapsto L(m, n)(1+\min (1 / L(i, j)-1, \lambda) \delta((m, n)-(i, j))) \\
& L_{-}(i, j):(m, n) \longmapsto L(m, n)(1-\min (1 / L(i, j)-1, \lambda) \delta((m, n)-(i, j)))
\end{aligned}
$$

where $\delta$ is the Dirac delta function.

Remark 1. By the use of the increment $k=\min (1 / L(i, j)-1, \lambda)$, we impose that the values of the images $L_{+}(i, j)$ do not exceed 1 . Moreover, the assumption $\lambda \in] 0,1\left[\right.$ imposes that the values of the images $L_{-}(i, j)$ do not become negative.

Then, we define the discrete version of the term (11) as

$$
\frac{\partial \operatorname{met}(L, H)(m, n)}{\partial L(i, j)}:=\frac{\operatorname{met}\left(L_{+}(i, j), H\right)(m, n)-\operatorname{met}\left(L_{-}(i, j), H\right)(m, n)}{2 \min (1 / L(i, j)-1, \lambda)}
$$

from which we derive each component $(i, j)$ of the discrete gradient:

$$
\frac{1}{M N} \sum_{m=1}^{M} \sum_{n=1}^{N} \partial \Phi\left(\operatorname{met}(L, H)(m, n) ; \frac{\left[\operatorname{met}\left(L_{+}(i, j), H\right)-\operatorname{met}\left(L_{-}(i, j), H\right)\right](m, n)}{2 \min (1 / L(i, j)-1, \lambda)}\right)
$$

Finally, we perform the gradient descent (6) where $\nabla(E)\left(L_{k}\right)$ is given by (10)) 


\section{Optimization of Tone Mapping Operators}

In this Section, we make use of the minimization approach of Sect. 2 in order to improve TMOs relatively to a given metric. This is a general approach which can be applied to any TMO. Whereas any dynamic range independent metric that can be defined as a non local metric in the sense of Def.1 (see Sect. 2.1) can be used, we focus in our experiments on the metric DRIM [3].

\subsection{Dynamic Range Independent Metrics (DRIM)}

The metric DRIM compares images of any dynamic range. For applications to tone mapping evaluation, the inputs are a HDR reference image $H$ and its LDR tone-mapped image $L$. The purpose of the metric is to consider the perception that a viewer would have of both scenes relying on psychophysical data, and to estimate at each pixel the probabilities that distortions appear. The distortions are based on the detection and classification of visible changes in the image structure. Three types of distortion are considered: Loss of Visible Contrast (LVC), contrast visible in the HDR image and not in the LDR image; Amplification of Invisible Contrast (AIC), details that appear in LDR image that were not in the HDR image; and contrast reversal (INV), contrast visible both in the HDR and LDR images with different polarity.

Each type of distortion can be represented as a $[0,1]$-valued function on the image domain $\Omega \subset \mathbb{R}^{2}$. Then, we define a metric met (in the sense of Def.1) between HDR and tone mapped LDR images by

$$
\operatorname{met}(L, H):=(L V C, A I C, I N V)
$$

from which we define the distance $E(L, H)$ between $L$ and $H$ associated to met as

$$
E(L, H)=\frac{1}{M N} \sum_{m=1}^{M} \sum_{n=1}^{N} \sqrt{L V C(m, n)^{2}+A I C(m, n)^{2}+I N V(m, n)^{2}}
$$

Note that the function $\phi$ of Def.2 we take in the expression of (12) is the Euclidean norm on $\mathbb{R}^{3}$. Applying formula (10) to the energy (12), we obtain the expression of the gradient of the functional $L \longmapsto E(L, H)$

$$
\nabla(E(L, H))(i, j)=\frac{1}{M N} \sum_{m=1}^{M} \sum_{n=1}^{N} \frac{\left\langle\operatorname{met}(L, H(m, n)), \frac{\partial \operatorname{met}(L, H)}{\partial L(i, j)}(m, n)\right\rangle}{\phi(\operatorname{met}(L, H)(m, n))}
$$

where the term $\frac{\partial m e t(L, H)}{\partial L(i, j)}$ is given by (9). Then we perform the gradient descent method (6) in order to reduce the distance (12) between the TM image $L$ and the HDR source $H$.

The existence of global extrema of the energy (12) is ensured. Indeed, the energy is bounded below by 0 and above by $\sqrt{3}$ since the metric met is bounded 
below by 0 and above by $\sqrt{3}$. Moreover, the set $L^{2}(\Omega ;[0,1])$ on which we minimize the energy is closed and bounded.

However, local extrema might exist because we do not have information about the convexity of the energy. Hence, the gradient descent method we apply may converge to a local minimum that is not global.

Remark 2. Even if the theoretical lower bound of the energy is 0, it is barely conceivable that this bound might be reached. Indeed, experiments show that we have $E(L, L)>0$ and $E(H, H)>0$ when we test the metric DRIM on any LDR image $L$ or HDR image $H$ of non constant intensity values. Hence, we claim that the situation $E(L, H)=0$ does not occur with the DRIM metric. As a consequence, we do not know if the critical point our algorithm reaches is a local or global minimum.

\subsection{Preprocessing}

To increase the chance that the algorithm converges to an image that has the smallest distance to $H$, i.e. a global minimum, we apply a preprocessing on the original TM image $L$ in order to get an initial condition of our algorithm that is closer (in terms of distance) to $H$ than $L$. The method we propose relies on the intuition that high values of LVC might be reduced by application of local sharpening whereas high values of AIC might be reduced by local Gaussian blurring. Hence we perform local Gaussian blurring and unsharp masking [1] to the initial LDR image depending on the values of the function $L V C-A I C$.

Denoting by $L_{\text {smooth }}$ a blurred version of $L$ and defining $L_{\text {sharp }}$ as

$$
L_{\text {sharp }}=L+\alpha\left(L-L_{\text {smooth }}\right)
$$

for some constant $\alpha$, we define the image $L_{n e w}$ as

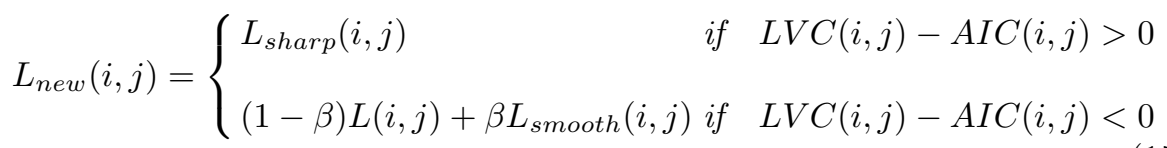

Experiments (see Table 1) show that we do have $E\left(L_{n e w}, H\right)<E(L, H)$.

\subsection{Experiments}

As the metric DRIM only takes into account the luminance information of the images, we first convert the LDR color image into a luminance map. It is performed by mapping its RGB values into the Lab color space and extracting the L-channel. Then inverse gamma correction is applied.

We perform the preprocessing described in Sect. 3.2 with the following parameters: the parameter $\sigma$ of the Gaussian smoothing kernel is set to 0.62 , and the constants $\alpha, \beta$ are respectively 0.7 and 0.5 . These values provide good results and have been fixed for all the experiments in this paper. 

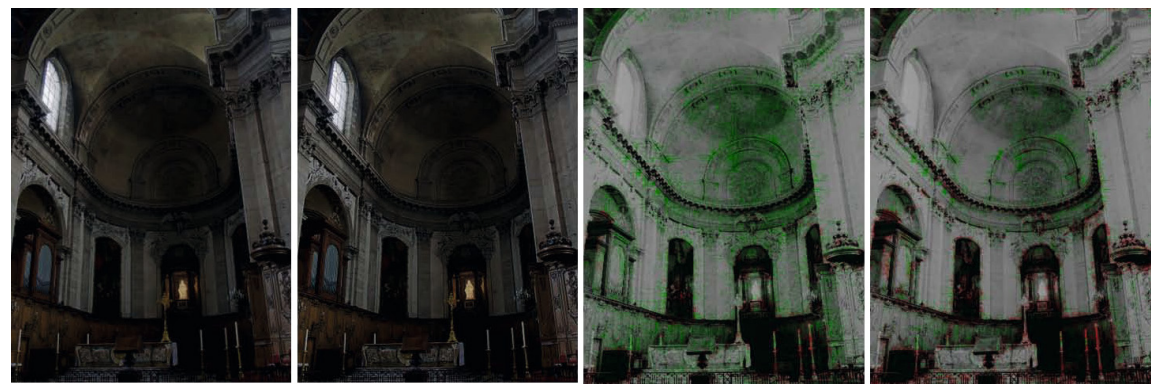

Fig. 1. The preprocessing stage. From left to right: input TM image (distance $=0.717$ ), output image (distance $=0.682$ ), distortion map of the input, distortion map of the output.In this case, the preprocessing reduces the TM error by $5 \%$.

Then, we apply the gradient descent algorithm of Sect. 3.1, where the initial condition is the output of the preprocessing. Because the variational problem we propose is non local, the gradient descent algorithm is very time-consuming. In order to decrease the execution time of the algorithm, rather than computing the sum in eq. (10) over the whole image, we only consider $50 \times 50$ neighbourhoods. The algorithm stops when a local minimum is reached, i.e. when the energy does not decrease anymore.

The output LDR color image is obtained by adding the components a,b of the initial TM image to the output LDR luminance map.

The evaluation of our algorithm is two-fold: global and pixel-wise. As a global measure, we consider the total energy (7) of the output (see Table1). As a pixelwise measure, we make use of the distortion map in [3. The basic idea is to encode contrast distortions by colors: green hue represents LVC, blue hue stands for AIC and red hue indicates INV, the saturation encoding the magnitude of the corresponding distortion, whereas the intensity corresponds to the intensity of the HDR source image (up to rounding). At each pixel, the maximum of the three distortions is computed, and the corresponding color is displayed. If the maximum is lower than 0.5 , then the saturation is set to 0 .

The first experiment consists in evaluating the preprocessing. In Fig. 11 we show an output color image of the preprocessing, as well as its distortion map. We have applied the formula (15) to a TM image obtained with the method of Ferradans et al. 9]. The HDR source image is taken from the MPI database [17. We observe that the LVC distortion has been reduced, whereas the INV distortion has increased a bit. As we can see in formula (15), the preprocessing is only devoted to reduce the LVC and AIC distortions, and does not take into account the INV distortion. This might explain why the INV distortion tends to increase. On Table 1, we present results of the preprocessing tested on images of the Fairchild database [7] for different TMOs, i.e. Ferradans et al. [9], Drago et al. [5], Reinhard et al. [20, Mantiuk et al. [15]. The images have been rescaled to $200 \times 200$ pixels in order to speed the algorithm up. Average results have been computed over 5 images of the dataset. The results confirm that the preprocessing reduces the distance with the HDR source image. 

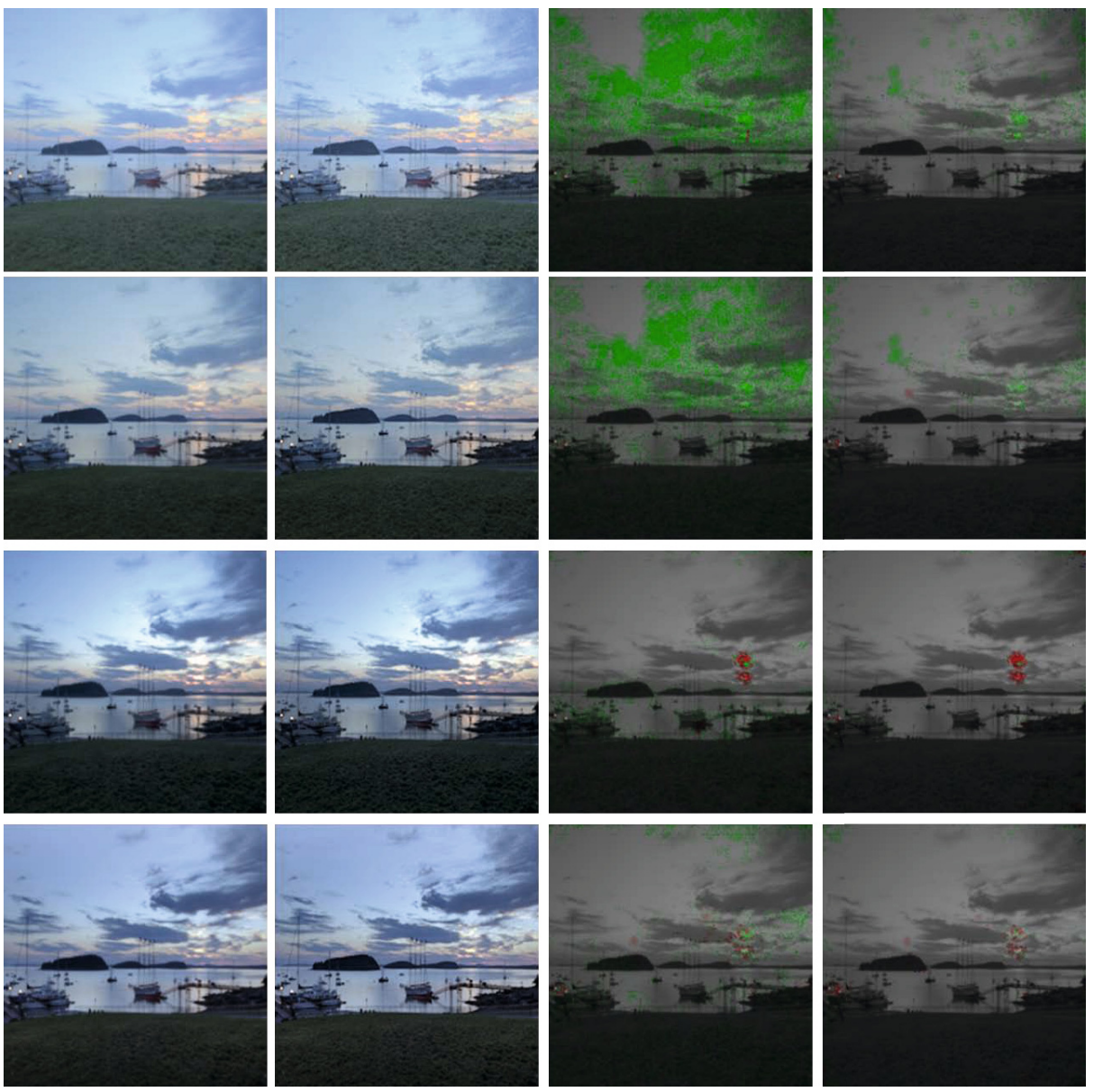

Fig. 2. The final output. First column: input TM images (from top to bottom: Drago et al. [5], Reinhard et al. 20, Mantiuk et al.[15], Ferradans et al. 9] TMOs). Second column: final output images. Third column: distortion maps of input TM images. Fourth column: distortion maps of final outputs. See Table. 1 (image "BarharborPresun") for the corresponding distances.

In the second experiment, we evaluate the final output of our method described above. In Fig. 2 we show some results of our algorithm tested on the different TMOs mentioned above. The HDR source is the image "BarharborPresun" taken from Fairchild database. The distortion maps show that the algorithm reduces drastically the distortion $\mathrm{LVC}$, but the INV tends to remain. Table 1 shows the distances of the initial TM images and output images with the HDR source image, as well as for other images of the Fairchild dataset. As expected, the final distance is lower than the initial distance. An other interesting point is the observation in Fig. 2 that the contrast in the output color images is higher than the one in the initial TM images. 

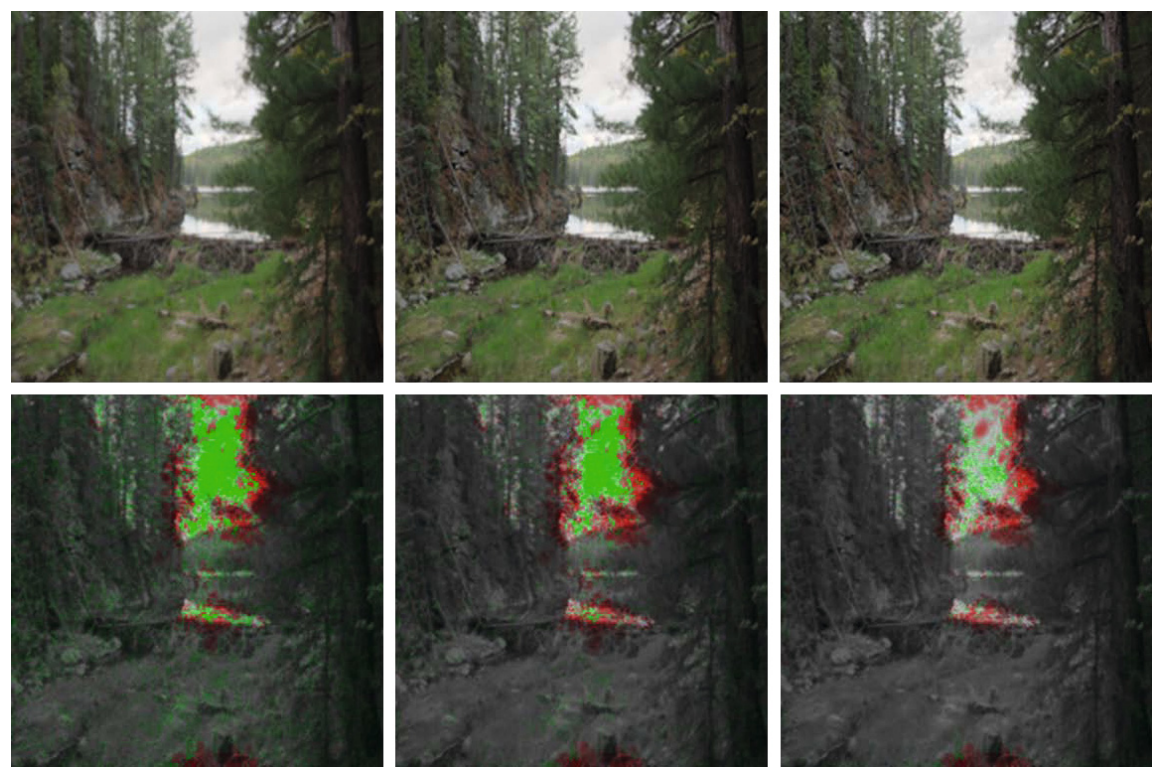

Fig. 3. Comparison between the preprocessing stage and the final output. Top left: input TM image (distance $=0.708$ ). Top middle: output of preprocessing stage (distance $=0.615)$. Top right: final output $($ distance $=0.533)$. Bottom row: corresponding distortion maps. For this image our method reduces the TM error by $25 \%$.

Table 1. Distance at each stage of our method computed with DRIM 3

\begin{tabular}{|c|c|c|c|c|c|}
\hline Image & TMO & Initial & Preprocessing & Final & Improvement(\%) \\
\hline \multirow{4}{*}{ BarharborPresun } & Drago et al. & 0.681 & 0.608 & 0.502 & 26.25 \\
\cline { 2 - 6 } & Reinhard et al. & 0.676 & 0.607 & 0.493 & 27.05 \\
\cline { 2 - 6 } & Mantiuk et al. & 0.528 & 0.487 & 0.424 & 19.75 \\
\cline { 2 - 6 } & Ferradans et al. & 0.560 & 0.509 & 0.438 & 21.46 \\
\hline \multirow{3}{*}{ AmikBeavDamPM1 } & Drago et al. & 0.767 & 0.682 & 0.584 & 23.90 \\
\cline { 2 - 6 } & Reinhard et al. & 0.708 & 0.615 & 0.533 & 24.70 \\
\cline { 2 - 6 } & Mantiuk et al. & 0.726 & 0.649 & 0.564 & 22.25 \\
\cline { 2 - 6 } & Ferradans et al. & 0.691 & 0.630 & 0.542 & 21.50 \\
\hline \multirow{2}{*}{ Average } & Drago et al. & 0.672 & 0.606 & 0.525 & 21.91 \\
\cline { 2 - 6 } & Reinhard et al. & 0.636 & 0.564 & 0.464 & 27.09 \\
\cline { 2 - 6 } & Mantiuk et al. & 0.619 & 0.564 & 0.493 & 20.40 \\
\cline { 2 - 6 } & Ferradans et al. & 0.609 & 0.566 & 0.491 & 19.39 \\
\hline
\end{tabular}

At last, we compare the output of the preprocessing stage with the final output of our method. In Fig. 3, we compare distortion maps. The HDR source is the image "AmikeusBeaverDamPM1" from the Fairchild database. The input TM image is provided by the TMO of Reinhard et al. [20. The corresponding distortion map shows a high loss of contrast. We observe that the preprocessing stage reduces it to a great extent, and the gradient descent algorithm applied to 
the output of the preprocessing reduces it more. However, we see that neither the preprocessing nor the gradient descent algorithm achieves to reduce the INV distortion. The results on Table 1 confirm that applying the gradient descent algorithm to the output of the preprocessing provides better results than applying only the preprocessing.

\section{Conclusion}

We proposed a variational method to reduce the distance between two images of any dynamic range based on a non local metric. Then, dealing with the dynamic range independent metric DRIM, we presented an application to TMOs optimization. We tested our method on different TMOs of the literature. Experiments confirmed that our method substantially reduces the perceptual distance with the HDR source image, providing an average improvement of more than $20 \%$ for several state of the art TMOs.

Current work is devoted to improve the preprocessing stage by taking into account the INV distortion. Whereas the gradient term was approximated on $50 \times 50$ neighbourhoods in our experiments, we intend to decrease the execution time of our algorithm in order to compute the whole gradient term, which will probably improve our results. Finally, because our approach is a generic method that can be applied to images of any dynamic range and any non local metric, other applications might be envisaged in the future.

Acknowledgement. The authors would like to express their utmost gratitude to Tunc Aydin, Karol Myszkowski, Rafal Mantiuk and Hans-Peter Seidel for their help. This work was supported by the European Research Council, Starting Grant ref. 306337, and by Spanish grants ref. TIN2011-15954-E and ref. TIN2012-38112.

\section{References}

1. Adams, J.E., Deever, A.T., Morales, E.O., Pillman, B.H.: Perceptually based Image Processing Algorithm Design. Perceptual Digital Imaging: Methods and Applications 6, 123 (2012)

2. Ashikhmin, M.: A Tone Mapping Algorithm for High Contrast Images. In: Proc. Eurographics Workshop Rendering, pp. 1-11 (2002)

3. Aydin, T.O., Mantiuk, R., Myszkowski, K., Seidel, H.-P.: Dynamic Range Independent Image Quality Assessment. In: Proc. ACM SIGGRAPH, pp. 1-10 (2008)

4. Daly, S.: The Visible Differences Predictor: An Algorithm for the Assessment of Image Fidelity. In: Watson, A.B. (ed.) Digital Images and Human Vision, pp. 179206. MIT Press (1993)

5. Drago, F., Myszkowski, K., Annen, T., Chiba, N.: Adaptive Logarithmic Mapping for Displaying High Contrast Scenes. Computer Graphics Forum 22(3), 419-426 (2003)

6. Durand, F., Dorsey, J.: Fast Bilateral Filtering for the Display of High Dynamic Range Images. In: Proc. ACM SIGGRAPH, pp. 257-266 (2002) 
7. Fairchild, M.: http://www.cis.rit.edu/fairchild/HDRPS/HDRthumbs.html

8. Fattal, R., Lischinski, D., Werman, M.: Gradient Domain High Dynamic Range Compression. In: Proc. ACM SIGGRAPH, pp. 249-256 (2002)

9. Ferradans, S., Bertalmío, M., Provenzi, E., Caselles, V.: An Analysis of Visual Adaptation and Contrast Perception for Tone Mapping. IEEE Trans. on Pattern Analysis and Machine Intelligence 33(10), 2002-2012 (2011)

10. Jobson, D., Rahman, Z., Woodell, G.: A Multiscale Retinex for Bridging the Gap between Color Images and the Human Observation of Scenes. IEEE Trans. Image Processing 6(7), 965-976 (1997)

11. Krawczyk, G., Myszkowski, K., Seidel, H.-P.: Lightness Perception in Tone Reproduction for High Dynamic Range Images. Computer Graphics Forum 24(3), 635-645 (2005)

12. Kuang, J., Johnson, G.M., Fairchild, M.: iCAM06: A Refined Image Appearance Model for HDR Image Rendering. J. Visual Comm. and Image Representation 18, 406-414 (2007)

13. Mantiuk, R., Daly, S., Myszkowski, K., Seidel, H.-P.: Predicting Visible Differences in High Dynamic Range Images - Model and its Calibration. In: Human Vision and Electronic Imaging X. SPIE Proceedings Serie, vol. 5666, pp. 204-214 (2005)

14. Mantiuk, R., Myszkowski, K., Seidel, H.-P.: A Perceptual Framework for Contrast Processing of High Dynamic Range Images. ACM Trans. Applied Perception 3(3), 286-308 (2006)

15. Mantiuk, R., Daly, S., Kerofsky, L.: Display Adaptative Tone Mapping. In: Proc. ACM SIGGRAPH, vol. 68 (2008)

16. Mantiuk, R., Kim, K.J., Rempel, A.G., Heidrich, W.: HDR-VDP-2: A Calibrated Visual Metric for Visibility and Quality Predictions in all Luminance Conditions. In: Proc. ACM SIGGRAPH, vol. 40 (2011)

17. MPI database, http://www.mpi-inf.mpg.de/resources/hdr/gallery.html

18. Pattanaik, S., Tumblin, J., Yee, H., Greenberg, D.: Time-Dependent Visual Adaptation for Fast Realistic Image Display. In: Proc. ACM SIGGRAPH, pp. 47-54 (2000)

19. Reinhard, E., Ward, G., Pattanaik, S., Debevec, P.: High Dynamic Range Imaging, Acquisition, Display, and Image-Based Lighting. Morgan Kaufmann (2005)

20. Reinhard, E., Devlin, K.: Dynamic Range Reduction Inspired by Photoreceptor Physiology. IEEE Trans. Visualization and Computer Graphics 11(1), 13-24 (2005)

21. Shen, J.: On the Foundations of Vision Modeling, I. Weber's law and Weberized TV (Total Variation) Restoration. Phys. D 175, 241-251 (2003)

22. Smith, K., Krawczyk, G., Myszkowski, K., Seidel, H.-P.: Beyond Tone Mapping: Enhanced Depiction of Tone Mapped HDR Images. Computer Graphics Forum 25(3), 427-438 (2006)

23. Tumblin, J., Turk, G.: Lcis: A Boundary Hierarchy for Detail-Preserving Contrast Reduction. In: Proc. ACM SIGGRAPH, pp. 83-90 (1999)

24. Wang, Z., Bovik, A.C.: A Universal Image Quality Index. IEEE Signal Processing Letters 9(3), 81-84 (2002)

25. Ward, G., Rushmeier, H., Piatko, C.: A Visibility Matching Tone Reproduction Operator for High Dynamic Range Scenes. IEEE Trans. Visualization and Computer Graphics 3(4), 291-306 (1997) 